\title{
A Comparison of Nested PCR Assay with Conventional Techniques for Diagnosis of Intestinal Cryptosporidiosis in AIDS Cases from Northern India
}

\author{
Beena Uppal, Ompal Singh, Sanjim Chadha, and Arun Kumar Jha \\ Department of Microbiology, Maulana Azad Medical College and Lok Nayak Hospital, Bahadur Shah Zafar Marg, \\ New Delhi 110002, India \\ Correspondence should be addressed to Arun Kumar Jha; dr_jhauc2k@yahoo.co.in
}

Received 6 August 2013; Accepted 29 October 2013; Published 12 January 2014

Academic Editor: Wej Choochote

Copyright (C) 2014 Beena Uppal et al. This is an open access article distributed under the Creative Commons Attribution License, which permits unrestricted use, distribution, and reproduction in any medium, provided the original work is properly cited.

\begin{abstract}
Cryptosporidiosis is a very important opportunistic infection and is responsible for significant morbidity and mortality in HIV/AIDS patients. Although current laboratory methods are generally considered adequate to detect high concentrations of oocysts, they fail to detect cases of cryptosporidiosis in many immunocompromised patients. The present study was done to determine the diagnostic efficacy of modified Ziehl-Neelsen (ZN), antigen detection ELISA, and a nested PCR assay for detection of Cryptosporidium in 58 adult AIDS cases with diarrhea from the ART clinic of Lok Nayak Hospital, New Delhi. Cryptosporidium was detected in 17 (29.4\%), 39 (67.3\%), and 45 (77.5\%) cases by modified ZN staining, antigen ELISA, and nested PCR assay, respectively. Taking nested PCR as the gold standard, specificity of both modified ZN staining and Cryptosporidium antigen detection ELISA was $100 \%$ while the sensitivity of the tests was $37.8 \%$ and $86.6 \%$, respectively. PCR was more sensitive than the other two diagnostic modalities but required a more hands-on time per sample and was more expensive than microscopy. PCR, however, was very adaptable to batch analysis, reducing the costs considerably. This assay can therefore have considerable advantages in the treatment of immunosuppressed individuals like AIDS patients, allowing their early diagnosis and decreasing the morbidity and the mortality.
\end{abstract}

\section{Introduction}

Cryptosporidium parvum is an enteric protozoan parasite with worldwide distribution which may inhabit the gastrointestinal tract of a wide variety of animals including humans $[1$, 2]. Infection with C. parvum in immunocompetent persons often results in asymptomatic or mild self-limited disease, but in HIV-infected patients, particularly those with low CD4 counts, infection may result in chronic or life-threatening diarrhea, or extraintestinal disease [3-6]. In developed countries, an estimated $14 \%$ of AIDS patients with diarrhea have $C$. parvum infection [3]. In developing countries, the parasite is reported in 24\% (range: $8.7-48 \%$ ) of HIV-seropositive adults and children while in India it has been reported in $2-60 \%$ of such patients [7-11].

In persons with AIDS, cryptosporidiosis is commonly a permanent diarrheal illness that leads to chronic malabsorption of fluids, nutrients, vitamins, and electrolytes with resulting wasting [12]. This warrants a prompt diagnosis and an early institution of specific therapy to reduce the morbidity associated with the disease. But over the years, detection of this protozoon has become a challenge. Cryptosporidiosis is most commonly diagnosed by identifying oocysts in the stool specimens of infected persons. The diagnostic difficulties arise from the fact that shedding of $C$. parvum oocysts is intermittent even in patients with massive diarrhea [13]. The number of oocysts present in the stool sample may not be adequate for detection and it is usually seen that oocysts are better recovered from watery stools than the formed samples [14]. Modified Ziehl-Neelsen (ZN) staining and fluorescein-tagged monoclonal antibody immunofluorescence (IF) staining techniques are the most commonly utilized diagnostic modalities for intestinal cryptosporidiosis. However the sensitivity of these tests (modified ZN and IF staining) for detecting C. parvum oocysts in human stools has been reported to be 10,000 oocysts per $g$ of watery stool, while in formed stools 50,000 or 500,000 oocysts per gram are required for a positive IF or modified ZN staining 
test, respectively [15]. Therefore, newer and more sensitive techniques are clearly needed to identify these oocysts in the stool specimens.

Coproantigen detection assays and PCR-based methods (nested PCR being more sensitive) have been reported to have a high diagnostic index in such cases. Antigen assays have an advantage of not requiring a skilled microscopist and their specificity has been reported to be high. However, variable sensitivities and specificities have been reported using different kits. The commercially available coproantigen detection ELISA formats use monoclonal antibodies (mAbs) which recognize different sets of surface epitopes and $\mathrm{mAbs}$ used in these ELISA kits may not react or react weakly with antigens of different Cryptosporidium species. In addition the cost of the test per sample has been reported to be much more than microscopic examination. Thus, ELISA appears to offer no increase in sensitivity over microscopy [16].

PCR based methods have been shown to be more sensitive than the conventional microscopic and immunological methods for detection of C. parvum in human feces [17]. Balatbat et al. have reported that the nested PCR assay can identify as few as 500 oocysts per g of stool, which represents a 100 -fold increase in sensitivity compared with that of the IF method [18]. This assay may therefore contribute to the identification of patients who are asymptomatic but harbor infection at a threshold below that detectable by the current diagnostic tests.

With the above background in mind, this study was conducted to compare the nested PCR assay for detection of C. parvum with the conventional modified ZN staining and antigen detection in stool specimens by ELISA in order to determine the usefulness and practicality of PCR-based methods for diagnosis of C. parvum in stool specimens of AIDS patients.

\section{Materials and Methods}

2.1. Study Population. Fifty eight adult ( $>18$ years of age) drug naive HIV seropositive subjects fulfilling the WHO case definition of AIDS with or without diarrhea were enrolled from the ART clinic of Lok Nayak Hospital, New Delhi, India. Only those cases with CD4 $\mathrm{T}$ lymphocyte counts $<200$ cells/ $\mu \mathrm{L}$ were recruited.

2.2. Study Design. A cross-sectional study was conducted from October 2008 to August 2010 on 58 adult HIV seropositive patients in whom the presence of $C$. parvum in the fecal specimens was detected by applying three different diagnostic modalities: modified ZN staining of stool smears, detection of C. parvum antigen in the stool by ELISA, and nested PCR for detecting C. parvum DNA in the stool sample. The role of nested PCR as a diagnostic modality for detecting C. parvum in the stool samples of AIDS patients was evaluated. After an informed consent from the patients, a detailed history regarding the personal details, sociodemographic characteristics, diarrheal episodes, associated signs and symptoms, history of drug intake, exposure history, and so forth were recorded on a pre designed performa. Stool specimens were requested from all the participants.

2.3. Specimen Collection and Transport. Fecal samples were collected in clean, wide-mouthed screw capped disposable plastic container and transported to the microbiology laboratory by the patients themselves on the same day avoiding any unnecessary delay.

2.4. Laboratory Processing of Specimens. On receipt of the fecal specimen in the laboratory, the sample was divided into two equal portions. From the first aliquot stool smears were prepared, heat fixed, and stained by the Kinyoun's (modified ZN stain) method. Second aliquot was stored at $-20^{\circ} \mathrm{C}$ to perform ELISA and PCR.

One portion of second aliquot of stool specimen was used to detect $C$. parvum antigen by using a commercial ELISA kit for stool samples (IVD Research Inc. CA, USA) according to manufacturer's instructions. This test is a double antibody sandwich in vitro immunoassay for the qualitative determination of $C$. parvum antigen in the feces.

Another portion of second aliquot was used for molecular test. DNA was extracted by using QIAmp DNA stool minikit (QIAGEN, Hilden, Germany) according to manufacturer's instructions. A nested PCR approach was used to amplify a 194 bp DNA fragment of C. parvum which is located on chromosome 8 and is specific for $C$. parvum. This segment of C. parvum DNA as well as the outer primers and the probe correspond to sequences described by Laxer et al. [19]. The PCR method of Mullis and Faloona was used for amplification and the reaction mixture was prepared by the PCR master mix (Fermentas, Canada) containing Taq DNA polymerase and the deoxyribonucleotide triphosphates. The following DNA primers were used:

(1) outer primers

$$
\begin{aligned}
& \text { BB-1 ( } 5^{\prime} \text {-CCGAGT T TGATCCAAAAGTTAC- } \\
& \text { GAA-3') } \\
& \text { BB-2 }\left(3^{\prime} \text {-ATGATTATTC CGTATACTCC-5' }\right) \text {, }
\end{aligned}
$$

(2) inner primers

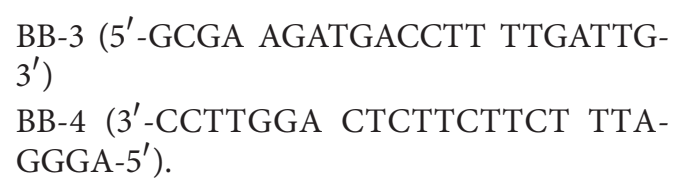

For the first amplification reaction the outer primers, BB-1 and BB-2, were used while for the second round of amplifications the inner primers, BB-3 and BB-4, were used The reactions were performed in a DNA thermal cycler (Mycycler, Bio-Rad, USA) [20]. Thermal-time profiles were the same as described by Balatbat et al. and amplification was done for 35 cycles [18]. Amplification products were visualized by electrophoresing the reaction mixture in $2 \%$ ethidium bromide stained agarose gel along with a molecular weight marker. Size markers included in all gels were the 1000 bp DNA ladder (Bio-basic Inc.) The electrophoresis was 
TABLE 1: C. parvum detected in study subjects by the various methods and the sensitivity, specificity, and predictive values of the modified ZN staining and antigen ELISA $(N=58)$.

\begin{tabular}{lccccc}
\hline Diagnostic technique & Subjects positive Number (\%) & Sensitivity & Specificity & Negative predictive & \multicolumn{2}{c}{ Positive predictive } \\
value & $100 \%$ & $100 \%$ & $31.7 \%$ \\
Modified ZN staining & $17(29.4)$ & $37.8 \%$ & $100 \%$ & $68.4 \%$ \\
C. parvum antigen ELISA & $39(67.3)$ & $86.6 \%$ & $100 \%$ & - & - \\
Nested PCR & $45(77.5)$ & - & - & - & - \\
\hline
\end{tabular}

TABLE 2: C. parvum positivity in study subjects by one or more of the diagnostic modalities $(N=58)$.

\begin{tabular}{lccc}
\hline $\begin{array}{l}\text { Modified ZN } \\
\text { staining }\end{array}$ & $\begin{array}{c}\text { C. parvum } \\
\text { antigen ELISA }\end{array}$ & Nested PCR & Total number (\%) \\
\hline+ & + & + & $17(29.3)$ \\
- & + & + & $20(34.5)$ \\
- & - & + & $7(12.1)$ \\
+ & - & - & 0 \\
+ & - & + & $1(1.7)$ \\
+ & + & - & 0 \\
- & + & - & $2(3.4)$ \\
- & - & - & $11(19.0)$ \\
\hline
\end{tabular}

carried out at a constant voltage of $90 \mathrm{~V}$ for 2 hours and a band of $194 \mathrm{bp}$ was taken to be positive result. The bands in the gel were photographed under UV transillumination.

2.5. Statistical Analysis. Sensitivity, specificity, positive predictive value, and negative predictive values of different diagnostic techniques were determined by taking nested PCR as the gold standard by using the statistical software SPSS version 17. $P$ values were calculated using the Fisher's Exact test.

\section{Results}

Of the 58 study subjects recruited, $44(75.86 \%)$ were male and $14(24.14 \%)$ were females with age ranging between 22 and 61 years (mean age $35.2 \pm 4.42$ years). The male to female ratio was 3.1:1. The predominant age group in males as well as females was $30-40$ years followed by 20 to 30 years. Most common mode of HIV acquisition was heterosexual contact (79.4\%) followed by injection drug abuse (10.3\%).

At the time of enrollment the mean CD4 T lymphocyte count of our study population was 129.2 cells $/ \mu \mathrm{L}$ and 45 out of 58 cases (77.5\%) had diarrhea. However all subjects had experienced an episode of diarrhea in the last one month prior to enrollment. Table 1 shows the $C$. parvum detected in study subjects by the various methods and the sensitivity, specificity, and predictive values of the modified ZN staining and antigen ELISA taking nested PCR assay as the gold standard and assuming it to be $100 \%$ sensitive and specific.

Table 2 shows the C. parvum positivity in study subjects by one or more of the diagnostic modalities. Seventeen samples $(29.3 \%)$ tested positive for C. parvum by all the three

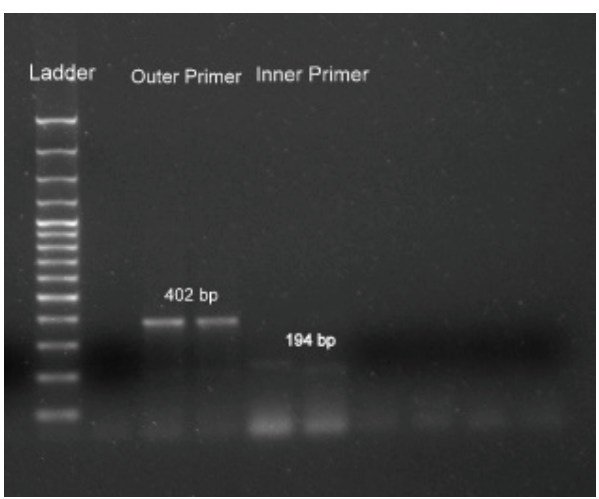

FIGURE 1: PCR product obtained with the first set of outer primers (402 bp) and the amplicon (194 bp) obtained after amplification with the second set of inner primers.

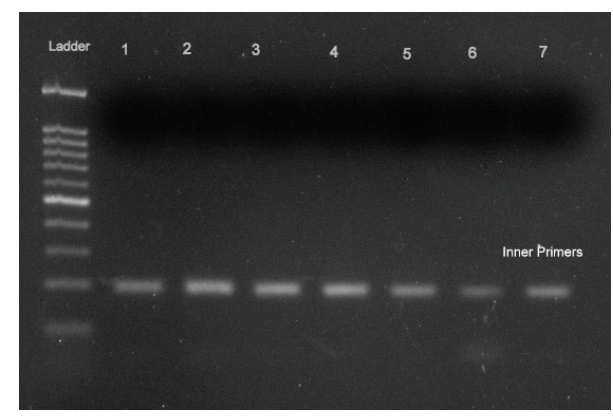

FIGURE 2: Nested PCR results with the inner primers yielded $194 \mathrm{bp}$ DNA fragment in the positive cases of Cryptosporidium. Lane 1: Positive control. Lanes 2-7: Amplicons of positive samples.

methods while 7 (12.1\%) samples were positive by nested PCR only. Two samples tested positive for C. parvum only by the antigen ELISA and not by the modified $\mathrm{ZN}$ staining and nested PCR assay.

Figure 1 shows a 402 bp PCR product obtained with the first set of outer primers and the $194 \mathrm{bp}$ amplicon obtained after amplification with the second set of inner primers from DNA extracted from oocysts present in positive fecal specimens.

Figure 2 shows the nested PCR results with the inner primers that yielded the expected $194 \mathrm{bp}$ DNA fragment in the stool specimens of positive cases of Cryptosporidium. Lane 1 is positive control and Lanes 2-7 are the amplicons of positive samples. 


\section{Discussion}

Although, with the widespread use of effective antiretroviral therapy (ART), cryptosporidiosis is no longer the devastating illness it once was in AIDS patients in developed countries, it still continues to pose a major threat to AIDS patients in resource-poor, developing countries like India where ART is not widely available or affordable. In patients with AIDS, intestinal cryptosporidiosis may sometimes be lethal and its diagnosis is therefore critically important. But some major pitfalls have been identified in the routine diagnosis of intestinal cryptosporidiosis [21]. Firstly, the direct microscopic examination after modified ZN staining relies on the morphologic recognition of small-sized oocysts which may be scant in number, intermittently shed, or inconsistently stained $[13,22]$. This method is therefore impractical to standardize as it is influenced by the individual skills of the microscopist involved. Furthermore the identification of an acid-fast blue-green alga (Cyanobacterium) which is only slightly larger than Cryptosporidium and has been associated with a prolonged self-limited diarrheal illness may limit the utility of acid-fast staining in the diagnosis of Cryptosporidium [23]. Secondly, the utility of the fluorescence based diagnostic tests is limited by expense and the frequent lack of a fluorescence microscope and trained staff in most of the diagnostic laboratories in developing countries like India. This technique also requires a more hands-on time per sample as batch testing is not possible [24]. C. parvum antigen testing by ELISA offers an alternative to the traditional microscopy based diagnostic tests as it is less time consuming and easier to perform and also enables the testing of a large number of samples at one time as batch testing can be undertaken. But the drawbacks associated with this test are the higher cost of the immunoassay kits and the specialist equipment (plate washers and readers) required to automate the whole process. Also the sensitivity and specificity of these tests have been reported to be lower than the immunofluorescence microscopy [25].

PCR technology offers a good alternative to conventional diagnosis of Cryptosporidium from both clinical as well as environmental samples [26]. The detection limits reported for PCR based methods by different authors have ranged from 100 to 2,000 oocysts per gram of human feces [27-29]. In this study we describe a sensitive and a specific nested PCR technique for detection of C. parvum directly in the stool specimens of AIDS patients.

The sociodemographic data of our study subjects was similar to that reported by some previous Indian studies done on HIV/AIDS cases with diarrhea, in particular the male preponderance, the most common age group of study subjects affected (sexually active young people) and the mode of HIV transmission (heterosexual route being the most common) $[7,30]$.

The study subjects in the present study (all of which were AIDS cases) had a mean CD4 level of 129.2 cells/ $\mu \mathrm{L}$, while Sehgal et al. reported the counts as $69.66 \pm 68.25$ cells $/ \mu \mathrm{L}$ and Ray et al. as $170 \pm 115$ cells $/ \mu \mathrm{L}$ in AIDS cases in India $[31,32]$. This discrepant observation can be attributed to the difference in the stage at which the subjects with AIDS were recruited for the study by the different investigators. $77.5 \%$ of our cases had diarrhea which is similar to what has been reported by National AIDS Control Organization in Delhi [33].

In our study specificity of both the techniques that is, modified $\mathrm{ZN}$ staining and $C$. parvum antigen detection ELISA was $100 \%$. Modified ZN staining had a sensitivity of $37.8 \%$ which is in accordance with previous studies where ZN staining has been found to be $98.9-100 \%$ specific with sensitivities ranging from 37-90\% [10, 26, 34]. C. parvum antigen detection ELISA in our study had a sensitivity of $86.6 \%$. Other investigators have reported sensitivities of $66.3-$ $100 \%$ and specificities of $93-100 \%$ using different kits for the antigen ELISA [16].

In the present study the agreement between microscopy and nested PCR showed that microscopy could identify $37.78 \%$ of the cases positive for C. parvum whereas ELISA diagnosed $82.22 \%$ C. parvum positive cases as compared to the nested PCR assay. Nested PCR assay was able to pick up $17.78 \%$ more positive cases as compared to modified $\mathrm{ZN}$ staining and antigen ELISA. These findings may be explained by the fact that direct microscopy relies on oocysts detection which might not be detectable in clinical samples from all cryptosporidiosis cases, and the absence of oocysts in repeated submissions of samples from symptomatic hosts does not necessarily indicate the absence of infection. In such cases, and particularly when clinical suspicion is high, antigen and/or PCR-based detection methods can be used, as sufficient C. parvum antigen or DNA from asexual life cycle forms is present in feces [35]. One of our study subjects had a positive direct microscopy and nested PCR while antigen ELISA was negative whereas two of our cases had a positive result for antigen ELISA and a negative result for modified ZN stain and nested PCR assay. Nonhomogeneous distribution of parasites in stool samples, lack of oocysts in the tested sample, and antigenic diversity among Cryptosporidium species explains the poor agreement among these three diagnostic modalities.

\section{Conclusions}

Nested PCR has the potential for accurate diagnosis in HIV seropositive subjects with diarrhea because of its high sensitivity. This will have considerable advantages in the treatment of AIDS patients, allowing early diagnosis before the onset of symptoms. Nested PCR test also has the added ability to directly differentiate between different Cryptosporidium genotypes, which assist in determining the source of cryptosporidial outbreaks. Sensitivity, specificity, ability to genotype, ease of use, and adaptability to batch testing make PCR a useful tool for future diagnosis and studies on the molecular epidemiology of Cryptosporidium infections. In spite of these advantages its wide spread use is still hindered by its high cost and it remains till now confined to research purposes and epidemiological studies. However there exists a valid explanation for this assay to be routinely used for C. parvum diagnosis. 


\section{Conflict of Interests}

The authors declare that there is no conflict of interests regarding the publication of this paper.

\section{Acknowledgments}

The authors would like to thank all the study subjects who volunteered and cooperated to become a part of this study. They also acknowledge the technical assistance provided by Mrs. Kamlesh Sharma. Their acknowledgement will not be complete without appreciating the role of their institution (Maulana Azad Medical College).

\section{References}

[1] B. A. Leav, M. Mackay, and H. D. Ward, "Cryptosporidium species: new insights and old challenges," Clinical Infectious Diseases, vol. 36, no. 7, pp. 903-908, 2003.

[2] L. Xiao, R. Fayer, U. Ryan, and S. J. Upton, "Cryptosporidium taxonomy: recent advances and implications for public health," Clinical Microbiology Reviews, vol. 17, no. 1, pp. 72-97, 2004.

[3] X. M. Chen, J. S. Keithly, C. V. Paya, and N. F. LaRusso, "Cryptosporodiosis," The New England Journal of Medicine, vol. 346, pp. 1723-1731, 2002.

[4] T. Flanigan, C. Whalen, J. Turner et al., "Cryptosporidium infection and CD4 counts," Annals of Internal Medicine, vol. 116, no. 10, pp. 840-842, 1992.

[5] F. J. Frost, K. Tollestrup, G. F. Craun, C. K. Fairley, M. I. Sinclair, and T. R. Kunde, "Protective immunity associated with a strong serological response to a Cryptosporidiumspecific antigen group, in HIV-infected individuals," Journal of Infectious Diseases, vol. 192, no. 4, pp. 618-621, 2005.

[6] I. Abubakar, S. H. Aliyu, C. Arumugam, N. K. Usman, and P. R. Hunter, "Treatment of cryptosporidiosis in immunocompromised individuals: systematic review and meta-analysis," British Journal of Clinical Pharmacology, vol. 63, no. 4, pp. 387-393, 2007.

[7] A. K. Jha, B. Uppal, S. Chadha et al., "Clinical and microbiological profile of HIV/AIDS cases with Diarrhea in North India," Journal of Pathogens, vol. 2012, Article ID 971958, 2012.

[8] O. Y. Bushen, A. Kohli, R. C. Pinkerton et al., "Heavy cryptosporidial infections in children in northeast Brazil: comparison of Cryptosporidium hominis and Cryptosporidium parvum," Transactions of the Royal Society of Tropical Medicine and Hygiene, vol. 101, no. 4, pp. 378-384, 2007.

[9] M. Joshi, A. S. Chowdhary, P. J. Dalal, and J. K. Maniar, "Parasitic diarrhoea in patients with AIDS," National Medical Journal of India, vol. 15, no. 2, pp. 72-74, 2002.

[10] K. Kaushik, S. Khurana, A. Wanchu, and N. Malla, "Evaluation of staining techniques, antigen detection and nested PCR for the diagnosis of cryptosporidiosis in HIV seropositive and seronegative patients," Acta Tropica, vol. 107, no. 1, pp. 1-7, 2008.

[11] S. V. Kulkarni, R. Kairon, S. S. Sane et al., "Opportunistic parasitic infections in HIV/AIDS patients presenting with diarrhoea by the level of immunesuppression," Indian Journal of Medical Research, vol. 130, no. 1, pp. 63-66, 2009.

[12] S. B. Mannheimer and R. Soave, "Protozoal infections in patients with AIDS: Cryptosporidiosis, isosporiasis, cyclosporiasis, and microsporidiosis," Infectious Disease Clinics of North America, vol. 8, no. 2, pp. 483-498, 1994.
[13] A. Clavel, A. C. Arnal, E. C. Sánchez et al., "Evaluation of the optimal number of faecal specimens in the diagnosis of cryptosporidiosis in AIDS and immunocompetent patients," European Journal of Clinical Microbiology \& Infectious Diseases, vol. 14, no. 1, pp. 46-49, 1995.

[14] W. L. Current and L. S. García, “Cryptosporidiosis," Clinical Microbiology Reviews, vol. 4, pp. 325-358, 1991.

[15] R. Weber, R. T. Bryan, H. S. Bishop, S. P. Wahlquist, J. J. Sullivan, and D. D. Juranek, "Threshold of detection of Cryptosporidium oocysts in human stool specimens: evidence for low sensitivity of current diagnostic methods," Journal of Clinical Microbiology, vol. 29, no. 7, pp. 1323-1327, 1991.

[16] S. Khurana, P. Sharma, A. Sharma, and N. Malla, "Evaluation of Ziehl-Neelsen staining, auramine phenol staining, antigen detection enzyme linked immunosorbent assay and polymerase chain reaction, for the diagnosis of intestinal cryptosporidiosis," Tropical Parasitology, vol. 2, pp. 20-23, 2012.

[17] T. Hanscheid, J. M. Cristino, and M. J. Salgado, "Screening of auramine-stained smears of all fecal samples is a rapid and inexpensive way to increase the detection of coccidial infections," International Journal of Infectious Diseases, vol. 12, no. 1, pp. 47-50, 2008.

[18] A. B. Balatbat, G. W. Jordan, Y. J. Tang, and J. Silva Jr., "Detection of Cryptospondium parvum DNA in human feces by nested PCR," Journal of Clinical Microbiology, vol. 34, no. 7, pp. 1769$1772,1996$.

[19] M. A. Laxer, B. K. Timblin, and R. J. Patel, "DNA sequences for the specific detection of Cryptosporidium parvum by the polymerase chain reaction," American Journal of Tropical Medicine and Hygiene, vol. 45, no. 6, pp. 688-694, 1991.

[20] K. B. Mullis and F. A. Faloona, "Specific synthesis of DNA in vitro via a polymerase-catalyzed chain reaction," Methods in Enzymology, vol. 155, pp. 335-350, 1987.

[21] S. Ogata, T. Suganuma, C. Okadac, K. Inoue, A. Kinoshita, and K. Sato, "A case of sporadic intestinal cryptosporidiosis diagnosed by endoscopic biopsy," Acta Medica Okayama, vol. 63, no. 5, pp. 287-291, 2009.

[22] F. G. Crawford and S. H. Vermund, "Human cryptosporidiosis," Critical Reviews in Microbiology, vol. 16, pp. 113-159, 1998.

[23] D. R. Shlim, M. T. Cohen, M. Eaton, R. Rajah, E. G. Long, and B. L. P. Ungar, "An alga-like organism associated with an outbreak of prolonged diarrhea among foreigners in Nepal," American Journal of Tropical Medicine and Hygiene, vol. 45, no. 3, pp. 383389, 1991.

[24] R. M. Chalmers, B. M. Campbell, N. Crouch, A. Charlett, and A. P. Davies, "Comparison of diagnostic sensitivity and specificity of seven Cryptosporidium assays used in the UK," Journal of Medical Microbiology, vol. 60, no. 11, pp. 1598-1604, 2011.

[25] T. Weitzel, S. Dittrich, I. Möhl, E. Adusu, and T. Jelinek, "Evaluation of seven commercial antigen detection tests for Giardia and Cryptosporidium in stool samples," Clinical Microbiology and Infection, vol. 12, no. 7, pp. 656-659, 2006.

[26] U. M. Morgan, L. Pallant, B. W. Dwyer, D. A. Forbes, G. Rich, and R. C. A. Thompson, "Comparison of PCR and microscopy for detection of Cryptosporidium parvum in human fecal specimens: clinical trial," Journal of Clinical Microbiology, vol. 36, no. 4, pp. 995-998, 1998.

[27] K. A. Webster, J. D. E. Pow, M. Giles, J. Catchpole, and M. J. Woodward, "Detection of Cryptosporidium parvum using a specific polymerase chain reaction," Veterinary Parasitology, vol. 50, no. 1-2, pp. 35-44, 1993. 
[28] P. Gobet, J. C. Buisson, O. Vagner et al., "Detection of Cryptosporidium parvum DNA in formed human feces by a sensitive PCR-based assay including uracil-N-glycosylase inactivation," Journal of Clinical Microbiology, vol. 35, no. 1, pp. 254-256, 1997.

[29] J.-R. Yu, S.-U. Lee, and W.-Y. Park, "Comparative sensitivity of PCR primer sets for detection of Cryptosporidium parvum," Korean Journal of Parasitology, vol. 47, no. 3, pp. 293-297, 2009.

[30] K. Ramakrishnan, R. Shenbagarathai, A. Uma, K. Kavitha, R. Rajendran, and P. Thirumalaikolundusubramanian, "Prevalence of intestinal parasitic infestation in HIV/AIDS patients with diarrhea in Madurai City, south India," Japanese Journal of Infectious Diseases, vol. 60, no. 4, pp. 209-210, 2007.

[31] S. Sehgal, U. Datta, S. Mujtaba, A. Sood, and V. K. Vinayak, "Cellular and serological markers of disease activity in Indian patients with HIV/AIDS," Methods in Cell Science, vol. 24, no. 1-3, pp. 107-114, 2002.

[32] K. Ray, S. M. Gupta, M. Bala, S. Muralidhar, and J. Kumar, "CD4/CD8 lymphocyte counts in healthy, HIV-positive individuals \& AIDS patients," Indian Journal of Medical Research, vol. 124, no. 3, pp. 319-330, 2006.

[33] S. N. Misra, D. Sengupta, and S. K. Satpathy, "Aids in India: recent trends in opportunistic infections," Southeast Asian Journal of Tropical Medicine and Public Health, vol. 29, no. 2, pp. 373-376, 1998.

[34] L. Tuli, D. K. Singh, A. K. Gulati, S. Sundar, and T. M. Mohapatra, "A multiattribute utility evaluation of different methods for the detection of enteric protozoa causing diarrhea in AIDS patients," BMC Microbiology, vol. 10, article 11, 2010.

[35] H. V. Smith, "Diagnostics," in Cryptosporidium and Cryptosporidiosis, R. Fayer and L. Xiao, Eds., pp. 173-208, CRC Press and IWA Publishing, 2nd edition, 2008. 

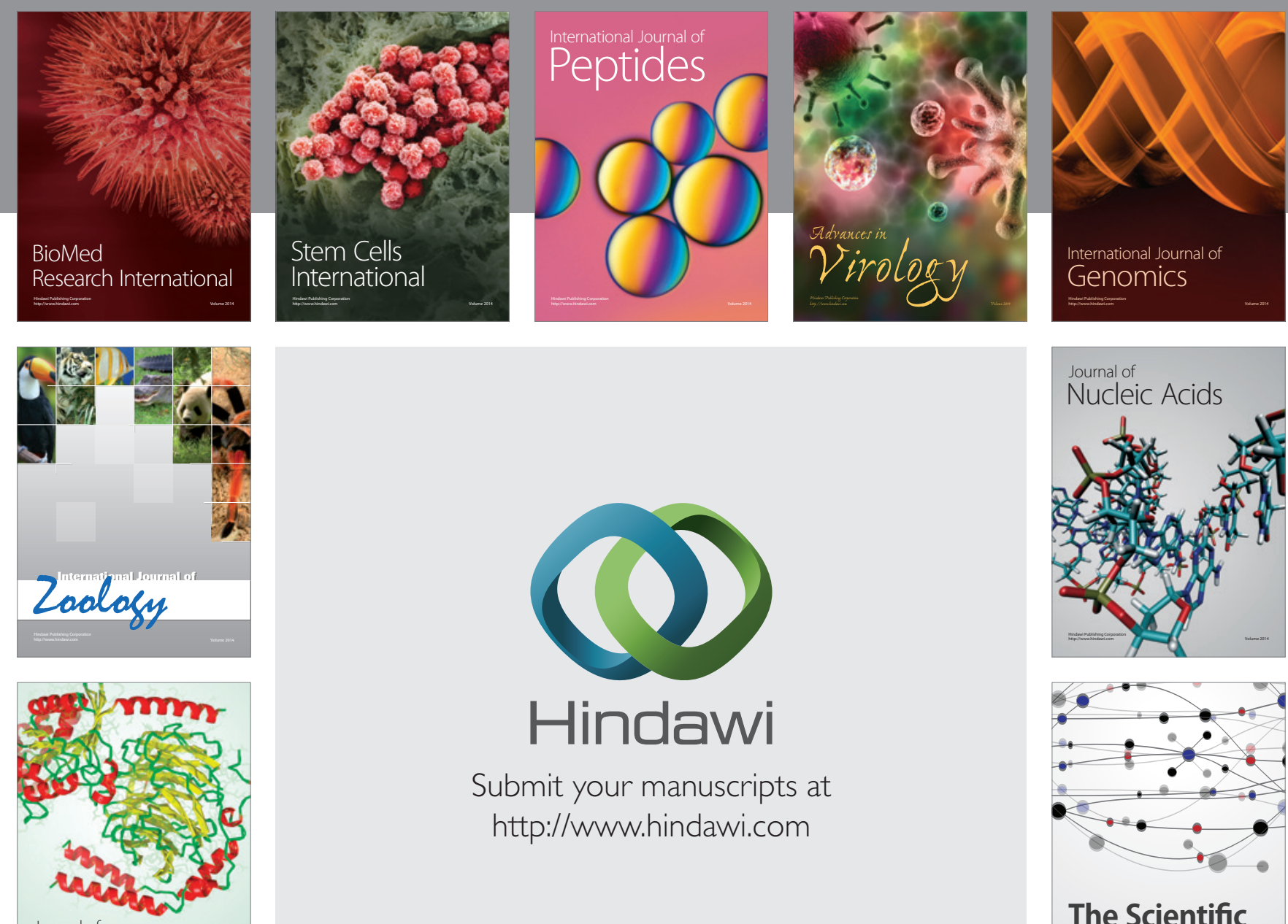

Submit your manuscripts at

http://www.hindawi.com

Journal of
Signal Transduction
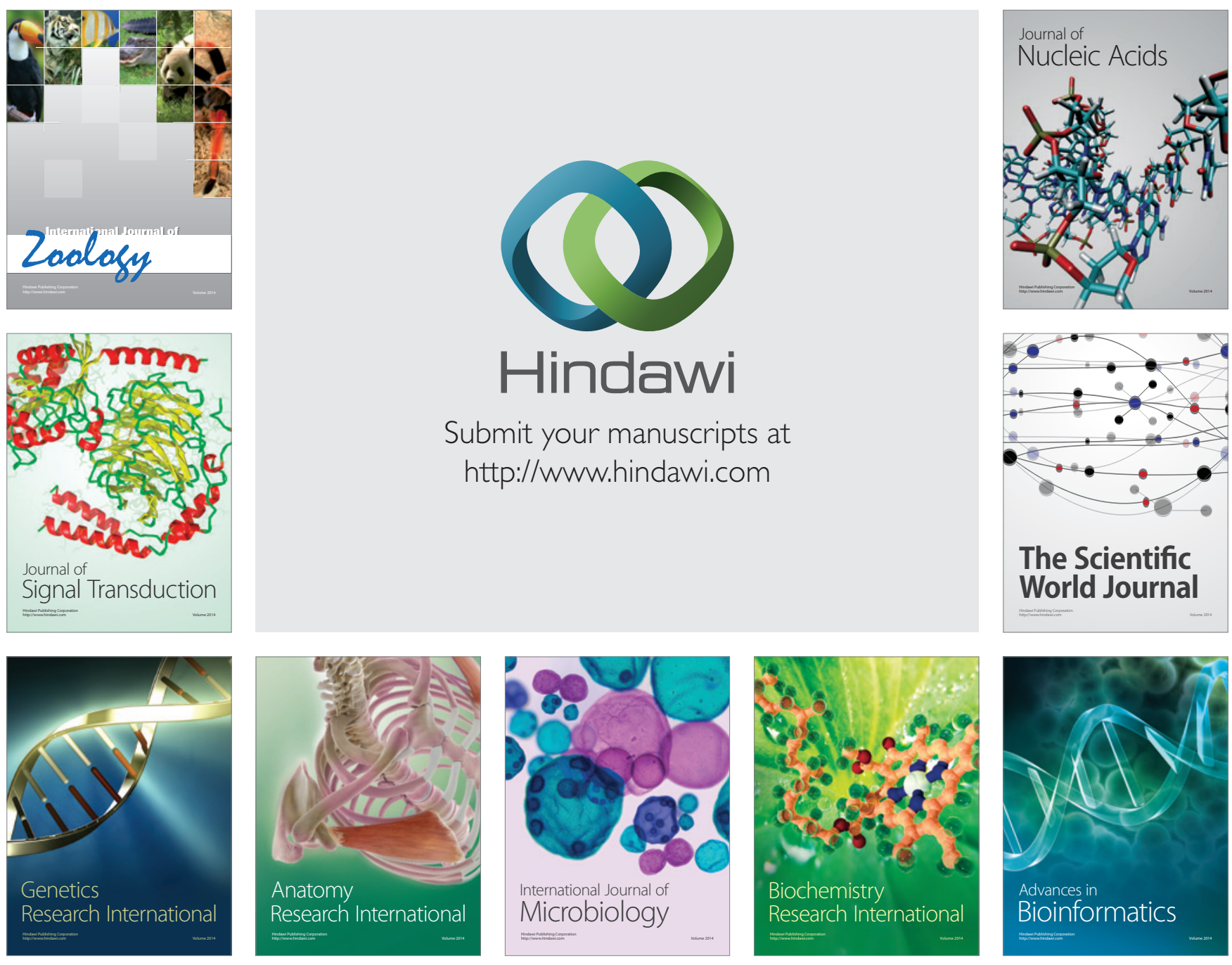

The Scientific World Journal
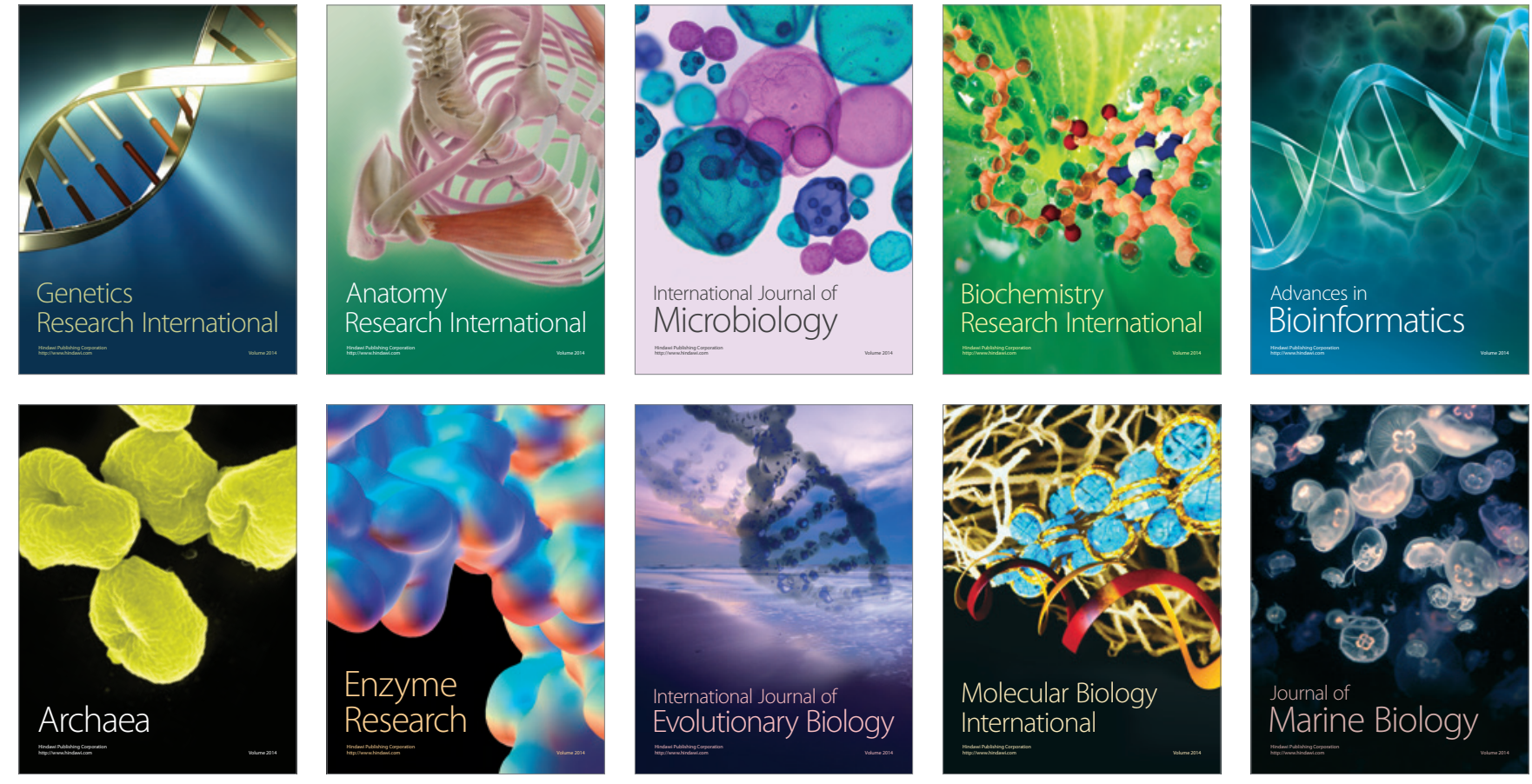